\title{
Transcriptional autoregulation of the RcsCDB phosphorelay system in Salmonella enterica serovar Typhimurium
}

\author{
María de las Mercedes Pescaretti, Fabián E. López, Roberto D. Morero \\ and Mónica A. Delgado
}

Correspondence

Mónica A. Delgado

monicad@fbqf.unt.edu.ar

Received 6 May 2010

Revised 20 July 2010

Accepted 13 August 2010

\begin{abstract}
Departamento de Bioquímica de la Nutrición, Instituto Superior de Investigaciones Biológicas (Consejo Nacional de Investigaciones Científicas y Técnicas-Universidad Nacional de Tucumán) and Instituto de Química Biologica 'Dr Bernabe Bloj', Chacabuco 461, 4000 San Miguel de Tucumán, Tucumán, Argentina
\end{abstract}

\section{INTRODUCTION}

The Rcs phosphorelay is an uncommon adaptive response system, composed of three proteins: the sensor RcsC, the cognate response regulator RcsB, and the intermediary in the phosphoryl transfer RcsD (Majdalani \& Gottesman, 2005). It has been determined that the flow of phosphoryl groups through the Rcs phosphorelay components occurs as follows: $\mathrm{RcsC} \rightarrow \mathrm{RcsD} \rightarrow \mathrm{RcsB}$ (Takeda et al., 2001). The Rcs system appears to be conserved in the family Enterobacteriaceae (Huang et al., 2006; Pescaretti et al., 2009), and it is involved in the modulation of the expression of many genes, such as those controlling colanic acid biosynthesis (Stout \& Gottesman, 1990), regulation of flagellum synthesis (Francez-Charlot et al., 2003), cell division (Carballes et al., 1999), O-antigen chain length determination (Delgado et al., 2006), motility (Cano et al., 2002) and Vi antigen synthesis (Virlogeux et al., 1996). The signals leading to induction of the Rcs system remain unknown, even though a wide range of activation conditions has been described, such as bacterial growth at low temperature or on solid surfaces (Ferrières \& Clarke, 2003), exposure to polymyxin B (Bader et al., 2003; Erickson \& Detweiler, 2006), overproduction of DjlA (Clarke et al., 1997; Chen et al., 2001; Kelley \& Georgopoulos, 1997), rcsC11 constitutive mutation (Costa \& Anton, 2001; Mouslim et al., 2004), igaA (Cano et al., 2002) and mucM mutants (Costa \& Anton, 2001), and tolB and pmrA mutants affecting the cell envelope (Mouslim \& Groisman, 2003).
Previously, we reported that the $\operatorname{rcs} B$ gene is transcribed from two promoters: (i) $\mathrm{P}_{r c s D B}$, located upstream of $r c s D$, and (ii) $\mathrm{P}_{r c s B}$, located within the $r c s D$ coding region, and that the overexpression of $r c s B$ decreases $r c s D$ transcription (Pescaretti et al., 2009). The discovery of $r c s D$ repression led us to investigate the potential role of RcsB in the mechanism of Rcs system regulation. In the present study, we demonstrated that high levels of the RcsB regulator control $r c s D$ expression by direct binding to the $\mathrm{P}_{r c s D B}$ promoter, negatively autoregulating the Rcs system. Rcs negative autoregulation was observed in an $r c s C 11$ mutant or after polymyxin B treatment, indicating the importance of the promoters in different physiological states. In addition, we also showed a physiological role in swarming behaviour repression for $\mathrm{P}_{r c s B}$, via the control of $r c s B$ expression.

\section{METHODS}

Bacterial strains, molecular techniques and growth conditions. Bacterial strains and plasmids used in this study are listed in Table 1. Phage P22-mediated transductions were used to introduce mutations into different genetic backgrounds, as described by Davis et al. (1980). Recombinant DNA techniques and bacterial growth at $37^{\circ} \mathrm{C}$ in Luria-Bertani (LB) medium were performed according to standard protocols (Sambrook et al., 1989). Kanamycin, ampicillin and chloramphenicol were used at final concentrations of $50 \mu \mathrm{g} \mathrm{ml}^{-1}$, $50 \mu \mathrm{g} \mathrm{ml}^{-1}$ and $25 \mu \mathrm{g} \mathrm{ml}{ }^{-1}$, respectively. 
Table 1. Bacterial strains and plasmids used in this study

\begin{tabular}{|c|c|c|}
\hline Strain or plasmid & Description* & Reference or source \\
\hline \multicolumn{3}{|c|}{ S. Typhimurium strains } \\
\hline $14028 s$ & Wild-type & Fields et al. (1986) \\
\hline EG12711 & $\Delta r c s B:: \mathrm{Cm}$ & This work \\
\hline EG14873 & $\operatorname{rcs} C 11$ & Mouslim et al. (2004) \\
\hline EG14539 & $\Delta r c s D:: l a c Z Y$ & Pescaretti et al. (2009) \\
\hline EG14932 & $\Delta r c s B:: \operatorname{lac} Z Y$ & Pescaretti et al. (2009) \\
\hline MDs1077 & $\Delta r c s D::$ lacZY $r c s C 11$ & This work \\
\hline MDs1017 & $\Delta \mathrm{P}_{r c s D B}:: \mathrm{Cm}$ & This work \\
\hline MDs1018 & $\Delta \mathrm{P}_{r c s B}:: \mathrm{Cm}$ & This work \\
\hline MDs1026 & $\Delta r c s B:: \operatorname{lac} Z Y \Delta \mathrm{P}_{r c s D B}:: \mathrm{Cm}$ & This work \\
\hline MDs1032 & $\Delta r c s B:: l a c Z Y \Delta \mathrm{P}_{r c s D B}::$ FRT & This work \\
\hline MDs1027 & $\Delta r c s B:: \operatorname{lac} Z Y \Delta \mathrm{P}_{r c s B}:: \mathrm{Cm}$ & This work \\
\hline MDs1034 & $\Delta r c s B:: \operatorname{lac} Z Y \Delta \mathrm{P}_{r c s B}::$ FRT & This work \\
\hline \multicolumn{3}{|l|}{ Plasmids } \\
\hline pUHE2-2lacI ${ }^{q}$ & $\operatorname{rep}_{\mathrm{pMB} 1} \mathrm{Ap}^{\mathrm{r}} \operatorname{lacl}^{q}$ & Soncini et al. (1995) \\
\hline $\operatorname{prcs} B$ & pUHE2-21 lacI $I^{q}$ containing $r c s B$ & Pescaretti et al. (2009) \\
\hline $\operatorname{prcs} D$ & pUHE2-21 lacl $^{q}$ containing $r c s D$ & This work \\
\hline pMS201 & Low copy vector for cloning promoters, pLtet01, derived from pZS21-luc, gfpmut2 & Beeston \& Surette (2002) \\
\hline $\mathrm{pP}_{r c S D B}$ & $\mathrm{Km}^{\mathrm{r}}$ pMS201 containing $122 \mathrm{bp}$ of $\mathrm{P}_{r c s D B}$ fused to gfpmut2 & Pescaretti et al. (2009) \\
\hline $\mathrm{pP}_{r c s B}$ & pMS201 containing 131 bp of $\mathrm{P}_{r c s B}$ fused to $g f p$ mut 2 & Pescaretti et al. (2009) \\
\hline
\end{tabular}

${ }^{\star}$ Gene designations are summarized by Sanderson et al. (1995).

Mutation of chromosomal promoters. The promoters $\mathrm{P}_{r c s D B}$ or $\mathrm{P}_{\text {rcsB }}$ were deleted from the chromosome of wild-type Salmonella enterica serovar Typhimurium (S. Typhimurium) strain 14028s using the one-step gene-inactivation method (Datsenko \& Wanner, 2000). Briefly, a chloramphenicol-resistance cassette $(\mathrm{Cm})$ was amplified from plasmid pKD3, using primers 4894 (5' ${ }^{\prime}$-CACGGTTATTCACTACACTCCCCTGCTCGACCGTGTAGGCTGGAGCTGCTTCG-3') and 4504 (5'-CGTTTCACATAACTGCTTGCCGGGTACCAGATTAAGCATGGCCATATGAATATCCTCCTTAG-3') for $\mathrm{P}_{r c s D B}$ deletion, and 2385 (5'-GCGTTGCTTTTACAGGTCGTAAACATAATGTAGGCTGGAGCTGCTTC-3') and 2386 (5'-GGCAATAATTACGTTCATATTGTTCATATGAATATCCTCCTTAG-3') for $\mathrm{P}_{r c s B}$ deletion. For $\mathrm{P}_{r c S B}$ deletion, the resulting PCR product was introduced into the region from nucleotide -219 upstream of $r c s D$ to nucleotide 2541 of the $r c s D$ coding sequence, leaving the $\mathrm{P}_{r c s B}$ promoter intact. On the other hand, for $\mathrm{P}_{r c s B}$ deletion, the resulting PCR product was introduced into the complete $r c s D$ coding sequence, leaving an undamaged $\mathrm{P}_{r c s D B}$ promoter (Fig. 2a). These mutations were introduced into strain EG14932, which contains an $\triangle r c s B::$ lacZY fusion, by P22-mediated transduction. To eliminate any polar effect, the chloramphenicol resistance cassette was removed using plasmid pCP20, as described by Datsenko \& Wanner (2000). Proper Cm removal was confirmed by direct nucleotide sequencing. The resulting strains, MDs1032 and MDs1034, respectively, were then transformed with plasmid prcsB.

$\boldsymbol{\beta}$-Galactosidase assays. Bacteria were grown to $\mathrm{OD}_{600} 0.2$ (about $2 \mathrm{~h})$ and then supplemented with IPTG $(0.35 \mathrm{mM})$, to overexpress $r c s B$ from the $\mathrm{P}_{\text {lac }}$ promoter of plasmid $\operatorname{prcs} B$, or with polymyxin $\mathrm{B}$ $\left(1 \mu \mathrm{g} \mathrm{ml}^{-1}\right)$, to induce the Rcs system. After growth for an additional $5 \mathrm{~h}$, the $\beta$-galactosidase activity was measured as described by Miller (1972). Control cultures were grown for $7 \mathrm{~h}$ in LB medium at $37{ }^{\circ} \mathrm{C}$ in the absence of IPTG or polymyxin B.

DNase I footprinting assay. DNase I protection assays were carried out using appropriately labelled primers, as described by Delgado et al. (2006). Fragments of DNA used for DNase I footprinting were amplified by PCR using chromosomal DNA from a wild-type $S$. Typhimurium strain (14028s) as template. Previously, primers 4136 (5'-TGCTTCGCATTCGGTTTTTTTTAC- $3^{\prime}$ ) and 4137 (5'-TGATCAGCAATAAGAAGAAACGGGT- $3^{\prime}$ ), which anneal to the coding and

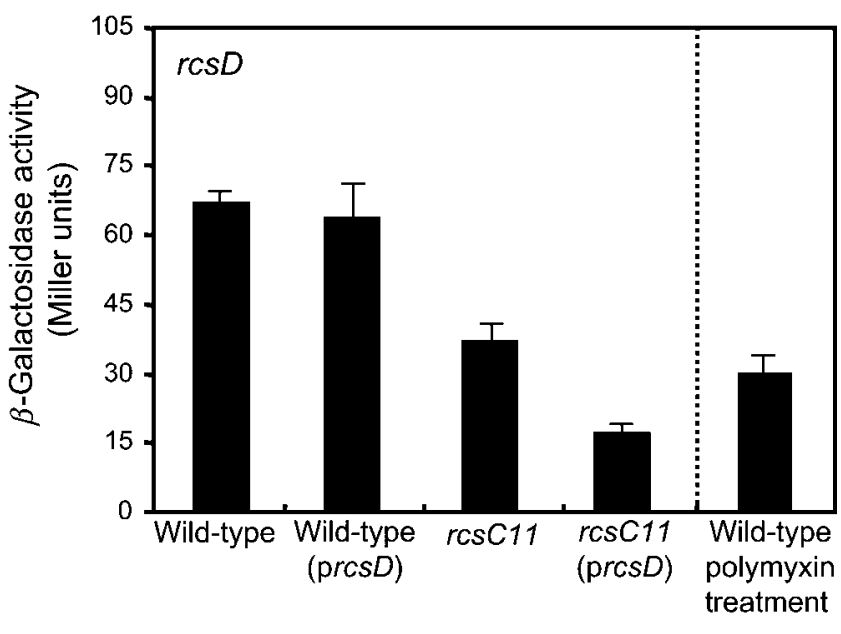

Fig. 1. $r \operatorname{cs} B$ overexpression represses $r c s D$ transcription. The transcriptional activity of a $\Delta r c s D:$ : lac $Z Y$ fusion, measured as $\beta$-galactosidase activity (Miller units), was investigated in the following genetic backgrounds: wild-type (EG14539) and rcsC11 (MDs1077), harbouring or not harbouring plasmid prcsD, and the wild-type (EG14539) strain in the presence of polymyxin B, as described in Methods. All data correspond to mean values of three independent experiments done in duplicate; error bars, SD. 

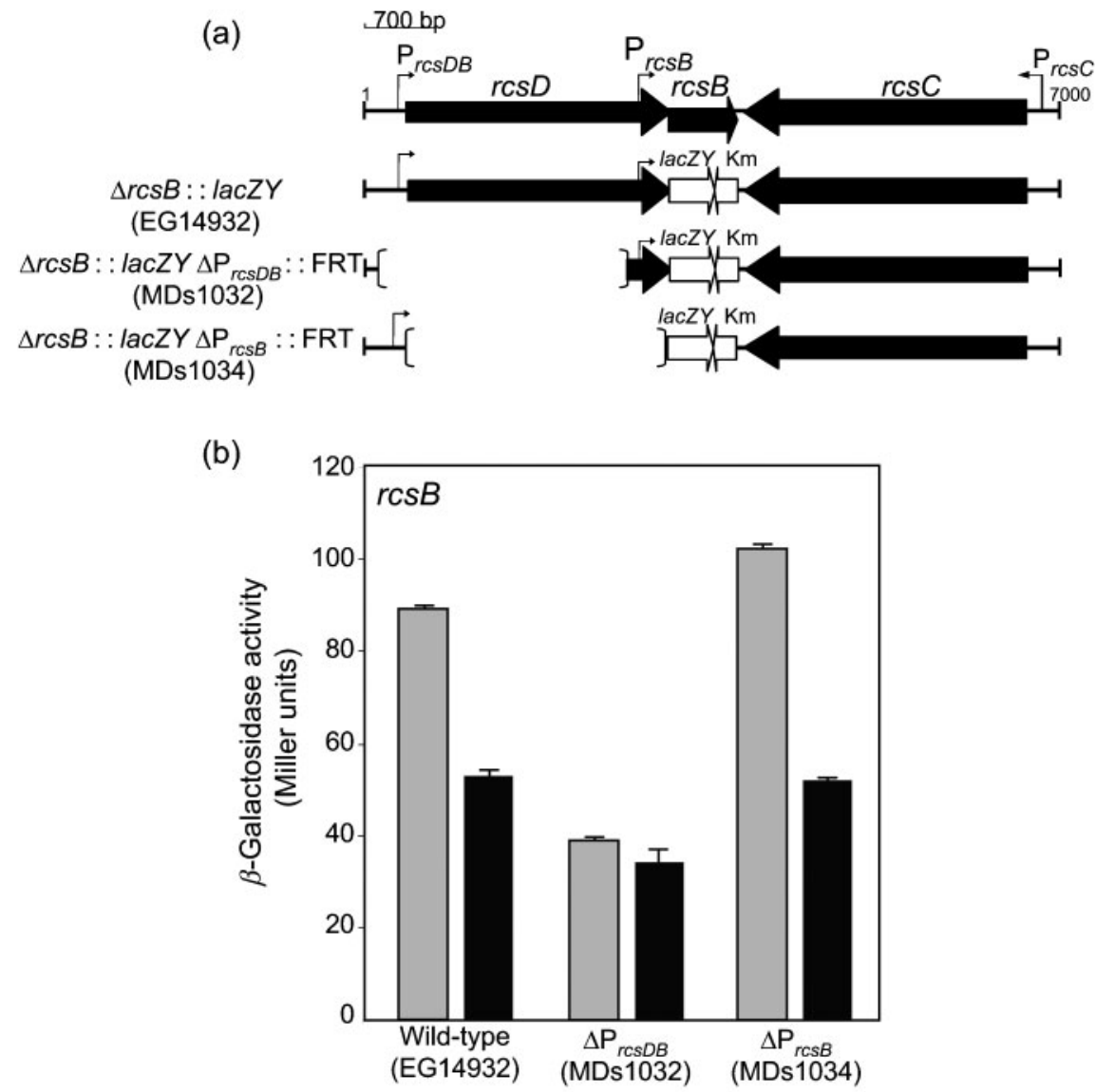

(c)

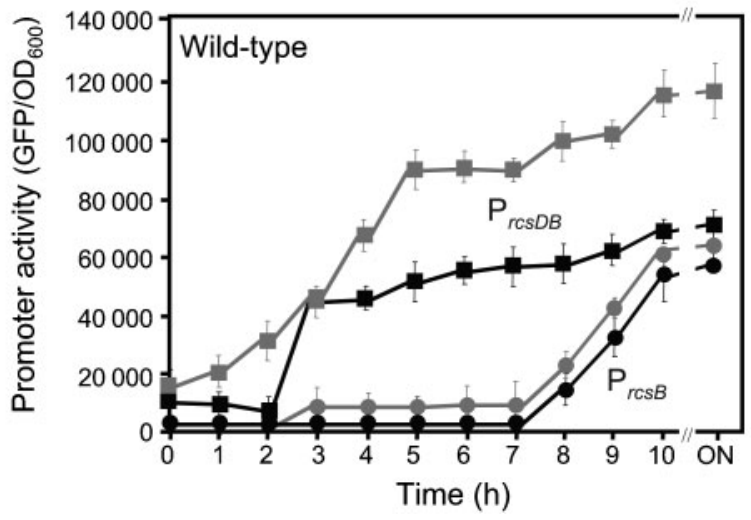

Fig. 2. Contribution of $\mathrm{P}_{r c s D B}$ and $\mathrm{P}_{r c s B}$ promoters to $r c s B$ expression. (a) Graphic representation of the genetic backgrounds used to determine the transcriptional activity of the $\operatorname{rcs} B$ gene. Spaces within brackets correspond to the deleted promoter regions. (b) Transcriptional activity of the $\triangle r c s B$ : lacZY fusion, measured as $\beta$-galactosidase activity (Miller units), was investigated in the following genetic backgrounds: wild-type (EG14932), and $\mathrm{P}_{r c s D B}$ (MDs1032) and $\mathrm{P}_{r c s B}$ (MDs1034) mutants, all carrying the prcsB plasmid and grown in the presence (black bars) or absence (grey bars) of IPTG. (c) $P_{r c s D B}$ (squares) and $P_{r c s B}$ (circles) promoter activity, measured as GFP production at each time point, was monitored in the wild-type 14028s strain co-transformed with $\mathrm{prcs} B$ and $\mathrm{pP}_{r c s D B}$ or $\mathrm{pP}_{r c s B}$ plasmids, respectively. Black symbols, cultures grown in the presence of IPTG; grey symbols, cultures grown in the absence of IPTG. All data correspond to mean values of three independent experiments done in duplicate; error bars, SD. 
non-coding strands of $r c s D$, respectively, had been labelled with T4 polynucleotide kinase and $\left[\gamma-{ }^{32} \mathrm{P}\right] \mathrm{ATP}$. The $r c s D$ promoter region was amplified with labelled primers 4136 and 4137 for the coding strand, or with labelled primers 4137 and 4136 for the non-coding strand. The histidine-tagged RcsB protein used in this work was purified as previously described (Delgado et al., 2006).

Determination of promoter activity by GFP production. As previously described (Pescaretti et al., 2009), wild-type S. Typhimurium (14028s) was transformed with plasmid pMS201, which contains a promoterless gfpmut2 gene in which the $\mathrm{P}_{r c s D B}$ or $\mathrm{P}_{r c s B}$ promoter region has been cloned. In this assay, promoter activity was measured as the rate of GFP production divided by the $\mathrm{OD}_{600}$ of the culture at each time point (Kalir et al., 2005; Ronen et al., 2002; Rosenfeld et al., 2002).

Swarming motility assay. Swarming assays were carried out as described by Kim \& Surette (2004). Briefly, the overnight LB cultures of tested strains were adjusted to $\mathrm{OD}_{600} 1.0$. Then, $5 \mu$ l of these normalized cultures was deposited onto the surface of $0.4 \%$ LB agar plates, which were incubated for $12 \mathrm{~h}$ at $37{ }^{\circ} \mathrm{C}$. To estimate the mean speed of migration $\left(\mathrm{mm} \mathrm{min}^{-1}\right)$, the diameter of migrating colonies $(\mathrm{mm})$ was plotted against the incubation time $(\mathrm{min})$. The images in Fig. 4 represent one of three independent experiments, while the data correspond to mean values for these independent experiments.

\section{RESULTS}

\section{RcsB overproduction represses rcs $D$ transcription}

We have previously demonstrated that $r c s B$ overexpression from plasmid $\mathrm{p} r c s B$ results in strong repression of $r c s D$ gene expression, while its own expression is not affected (Pescaretti et al., 2009). The differential expression of $r c s D$ and $r c s B$ is due to the presence of a second promoter, $\mathrm{P}_{r c s B}$, which activates $r c s B$ expression independently of $r c s D$ (Pescaretti et al., 2009). To determine whether the RcsB repressor effect is also produced under other Rcs-induction conditions, the $r c s C 11$ constitutive allele mutant was used. $r c s D$ expression levels, measured as the $\beta$-galactosidase activity of the chromosomal $\triangle r c s D$ : : lacZY fusion from wild-type $S$. Typhimurium (14028s) and the $r c s C 11$ mutant, were determined after $7 \mathrm{~h}$ of growth on LB medium. As shown in Fig. 1, the transcription level of $r c s D$ in the $r c s C 11$ mutant was twofold lower than that observed in the wildtype strain. Interestingly, a remarkable decrease (fourfold) was also observed in the mutant containing plasmid $\operatorname{prcs} D$ (Fig. 1). This result suggests that the phosphorylated RcsB is more effective than the non-phosphorylated form, due to the presence of the intermediary RcsD, which completes the Rcs phosphorelay pathway (Takeda et al., 2001). In a second approach, polymyxin B was used to induce the Rcs system. The data indicate that after $5 \mathrm{~h}$ of exposure to polymyxin B, the wild-type strain also showed twofold decreased levels of $r s c D$ expression (Fig. 1). These results suggest that $r c s D$ expression is repressed when the bacteria reach high levels of the RcsB regulator, under different Rcs system induction conditions.

\section{rcsB overexpression represses $P_{\text {rcsDB }}$ activity}

To determine whether high levels of RcsB affect $\mathrm{P}_{r c s D B}$ or $\mathrm{P}_{r c s B}$ promoter activity, we studied $r c s B$ expression in mutants with a deletion in each corresponding promoter region, $\mathrm{P}_{r c s D B}$ and $\mathrm{P}_{r c s B}$, transformed with $\mathrm{p} r c s B$. As shown in Fig. 2 (b), the $\beta$-galactosidase activity decreased 1.6-fold when $r c s B$ was overexpressed in the wild-type background compared with the corresponding control without $r c s B$ overexpression. Similarly, a 1.9-fold decrease was obtained with the $\mathrm{P}_{r c s B}$ mutant. Additionally, essentially no changes were observed when the overexpression was induced in the $\mathrm{P}_{r c s D B}$ mutant. These results clearly suggested that the $\mathrm{P}_{r c s D B}$ but not the $\mathrm{P}_{r c s B}$ promoter is repressed by high levels of RcsB. (a)

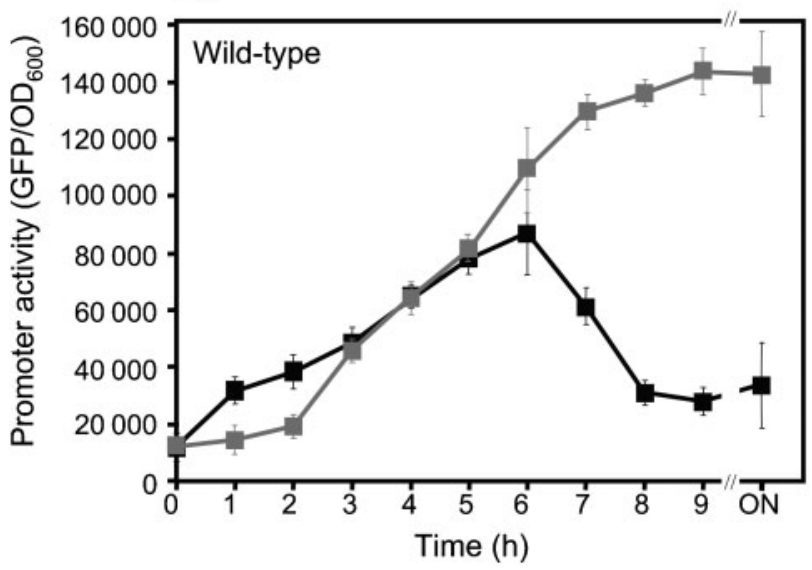

(b)

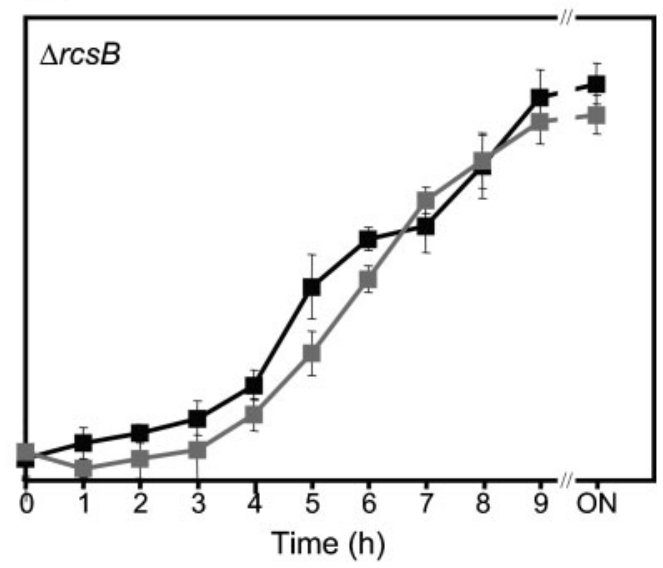

Fig. 3. $P_{r c s D B}$ activity is repressed by polymyxin $B$ treatment. $P_{r c s D B}$ promoter activity, measured as GFP production at each time point, was monitored in (a) wild-type strain 14028s and (b) the rcsB mutant (EG12711). Black symbols, cultures grown in the presence of polymyxin B; grey symbols, cultures grown in the absence of polymyxin B. All data correspond to mean values of three independent experiments done in duplicate; error bars, SD. 
(a) $\begin{array}{rr}P_{r c s D B}-(\mathrm{Se}) & \text { TACGTAAGAGTCTGGAAATT } \\ \text { ugd }+(\mathrm{Se}) & \text { GAATGAAGATAATCTGAATT } \\ \text { ams }+(\mathrm{Ea}) & \text { A ATTGAGAATAATCTTAATT } \\ \text { fts }+(\mathrm{Ec}) & \text { GAATTGAAGATCATCTGGTT }\end{array}$

(b)
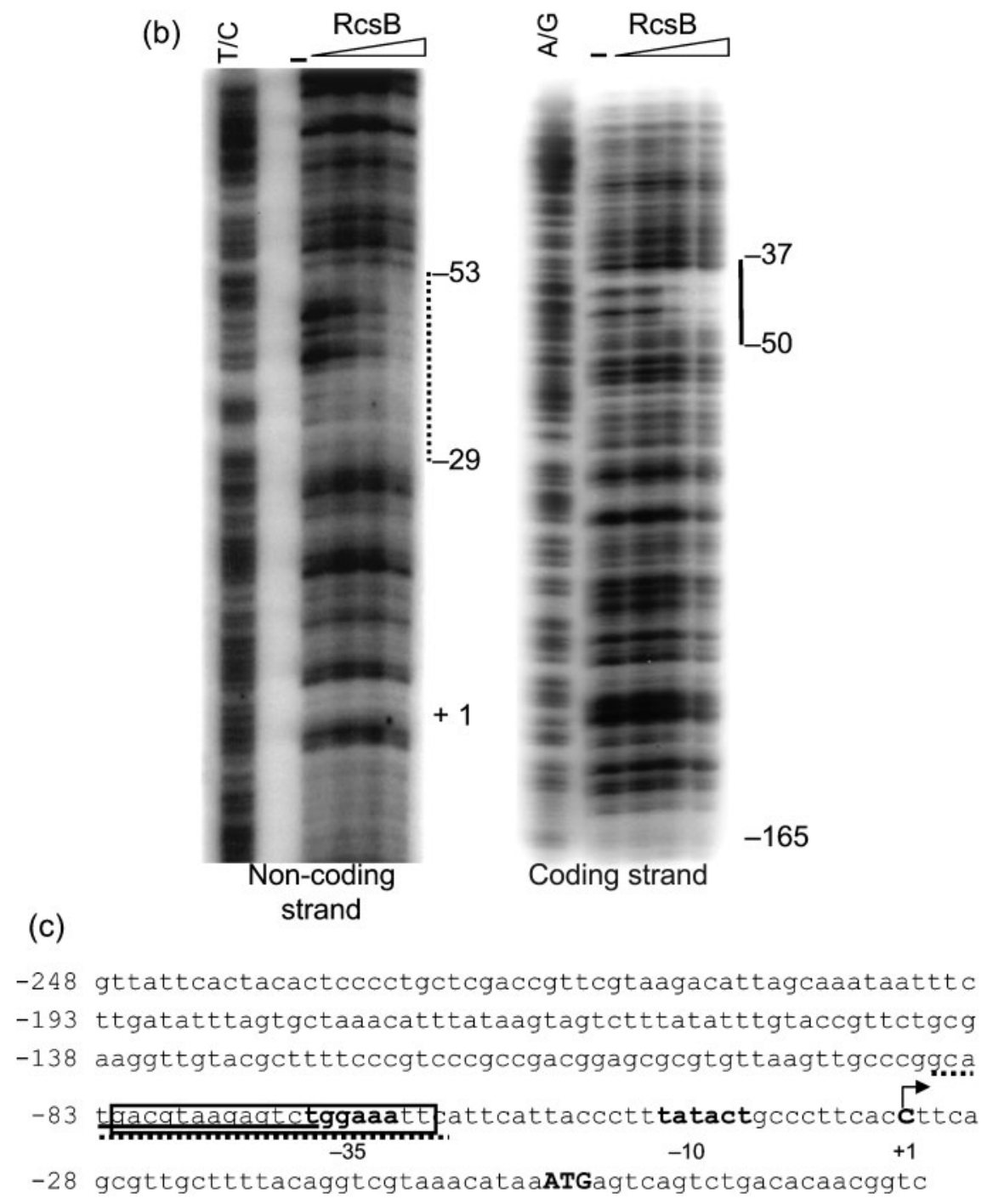

(c)

Fig. 4. Interaction of the RcsB regulator with the $P_{r c s D B}$ promoter region. (a) Alignment of the RscB boxes conserved in the $\mathrm{P}_{r c s D B}$ promoter in comparison with the reported RcsB-binding sequences of the S. Typhimurium (Se) ugd, Erwinia amylovora (Ea) ams, and E. coli $(\mathrm{Ec})$ fts genes. The box indicates the conserved sequence of the RcsB-binding motif. (b) DNase footprinting analysis of RcsB-His6 binding to the $\mathrm{P}_{r c s D B}$ promoter region. DNA footprinting analysis was performed on endlabelled fragments corresponding to the upstream $r \operatorname{cs} D$ coding and non-coding strands. The RcsB-His 6 protein was added at final concentrations of $0,10,40$ and $80 \mathrm{nM}$. Solid and dotted black bars represent the RcsB-protected regions. Lanes: A/G and T/C, Maxam and Gilbert sequencing reaction of the labelled fragments. (c) DNA sequence corresponding to the 248 bp region upstream of the $r c s D$ ORF. The sequences underlined by solid and dotted black lines represent the DNA regions footprinted by the RcsB-His6 protein. Conserved sequences corresponding to the putative RcsB-binding motif are boxed.

On the other hand, we expected that $\mathrm{P}_{r c s B}$ promoter activity would be absent and that $r c s B$ expression would be completely abolished by $\mathrm{P}_{r c s D B}$ deletion, under the experimental conditions employed (Fig. 2b). However, the $r c s B$ expression levels decreased by only $60 \%$. This was an unexpected result and could be explained by assuming that in the absence $\left(\mathrm{P}_{r c s D B}\right.$ mutant) or repression (wild-type strain overexpressing $r c s B$ ) of $r c s D$, the $\mathrm{P}_{r c s B}$ promoter activity is induced through an unknown mechanism, in order to maintain basal levels of $r c s B$ expression. We 
investigated this possibility, and the decreased levels of $r c s B$ expression, obtained when the $\mathrm{P}_{r c s D B}$ mutant was complemented with the prcsD plasmid, confirmed our assumption (data not shown).

To confirm that $\mathrm{P}_{r c D B}$ alone is repressed by high levels of RcsB and to simultaneously discount any polar effect, the $\mathrm{P}_{r c s D B}$ and $\mathrm{P}_{r c s B}$ activities were determined as GFP production. As shown in Fig. 2(c), $r c s B$ overexpression in the wild-type strain decreased the level of GFP when it was under the control of the $\mathrm{P}_{r c S D B}$ promoter. It is interesting to note that the repressive effect was observed only after $4 \mathrm{~h}$ of incubation. In contrast, no effect was detected when $\mathrm{P}_{\text {rcsB }}$ controlled the expression of GFP (Fig. 2c). The similarity of the results obtained with $\beta$-galactosidase activity and GFP expression assays let us conclude that high levels of $r c s B$ repress $\mathrm{P}_{r c s D B}$ activity, resulting in lower levels of $r c s D$ expression. The $\mathrm{P}_{r c s D B}$ promoter activity determined as GFP level was also measured after polymyxin B treatment. The exposure to polymyxin $\mathrm{B}$ of the wild-type strain transformed with plasmid pMS201 containing the $\mathrm{P}_{r c s D B}$ promoter region (Pescaretti et al., 2009) decreased GFP production compared with the control without antibiotic (Fig. 3a). This effect was not observed in the $r c s B$ mutant background (Fig. 3b), highlighting the role of the RcsB regulator. These results confirm our supposition that the effect of $r c s D$ repression occurs at the physiological levels of Rcs system induction.

\section{RcsB protein binds to the $\mathbf{P}_{\text {rcsDB }}$ promoter}

With the aim of demonstrating a direct repression effect of RcsB, we searched by bioinformatics analysis a putative RcsB-binding site on the $\mathrm{P}_{r c s D B}$ promoter region sequence. This analysis revealed the presence of a DNA sequence that exhibits homology with the previously predicted RcsBbinding box (Fig. 4a) (Carballes et al., 1999; Mouslim et al., 2003; Wehland \& Bernhard, 2000). DNase I footprinting assay of the $248 \mathrm{bp}$ region upstream of the $r c s D$ coding sequence established that the RcsB protein binds to the characterized $\mathrm{P}_{r c s D B}$ promoter (Pescaretti et al., 2009). Specifically, the region from position -50 to -37 on the coding strand and from -53 to -29 on the non-coding strand relative to the transcription start site was protected by the RcsB regulator (Fig. $4 \mathrm{~b}$ ). The protected sequences included the predicted RcsB-binding box and overlapped with the $\mathrm{P}_{r c s D B}$ promoter -35 box (Fig. $4 \mathrm{c}$ ).

\section{Swarming is controlled by the rcsB gene expressed under the $\mathbf{P}_{\text {rcsB }}$ promoter}

The swarming modulation of Escherichia coli and $S$. Typhimurium has been associated with the Rcs phosphorelay system (Harshey, 2003; Takeda et al., 2001; Toguchi et al., 2000). Here, we studied the motility phenotype of the wild-type $S$. Typhimurium strain, and of $r c s B, \mathrm{P}_{r c s D B}$ and $\mathrm{P}_{r c s B}$ mutants. The absence of a chromosomal $r c s D$ gene in the $\mathrm{P}_{r c s D B}$ and $\mathrm{P}_{r c s B}$ mutants was complemented with the prcs D plasmid, in order to complete the phosphorylation pathway and produce the more active form of RcsB (phospho-RcsB) (Mariscotti \& Garcia-del Portillo, 2009). In order to unify the genetic background, the wild-type $S$. Typhimurium strain and $r c s B$ mutant were also transformed with $\operatorname{prcs} D$. It is important to note that this assay was carried out in strains harbouring the chromosomal $r c s B$ gene and under growth conditions different from those used in Fig. 2(b). In agreement with previous observations (Delgado et al., 2006), the wild-type strain and the $r c s B$ mutant containing plasmid prcs $D$ displayed very different swarming behaviours, migrating at $1.8 \times 10^{-2}$ and $2.2 \times 10^{-2} \mathrm{~mm}$ $\min ^{-1}$, respectively (Fig. 5). Interestingly, the $\mathrm{P}_{r c s B}$ mutant migrated appreciably faster $\left(2.0 \times 10^{-2} \mathrm{~mm} \mathrm{~min}^{-1}\right)$ than the wild-type strain $\left(1.7 \times 10^{-2} \mathrm{~mm} \mathrm{~min}^{-1}\right)$ and $\mathrm{P}_{r c s D B}$ mutant $\left(1.6 \times 10^{-2} \mathrm{~mm} \mathrm{~min}^{-1}\right)$ (Fig. 5). This result could be explained by assuming that in the $\mathrm{P}_{r c s B}$ mutant, the RcsB levels produced exert a negative autoregulation on $\mathrm{P}_{r c s B}$ promoter activity, resulting in less repression of motility. In contrast, the negative autoregulation effect on the $\mathrm{P}_{r c s D B}$ promoter was not observed in the results obtained in Fig. 2(b) due to the absence of the $r c s B$ gene.
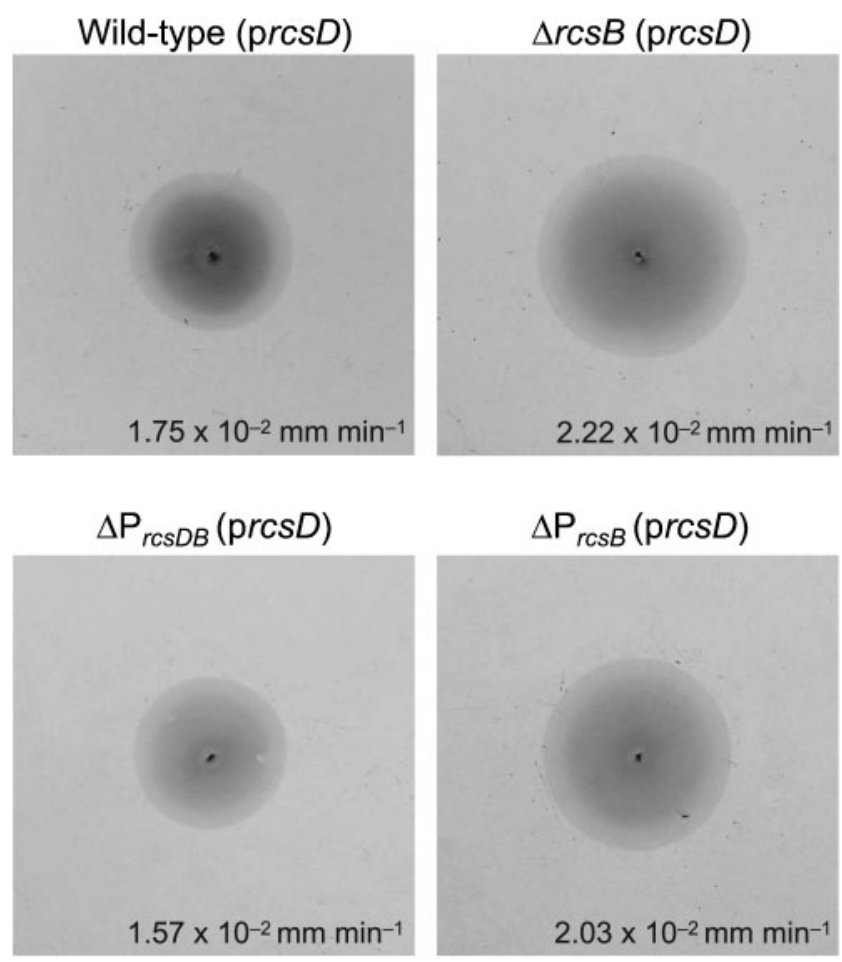

Fig. 5. $r c s B$ gene expression determines the swarming phenotype under the control of $\mathrm{P}_{r c s B}$. The swarming behaviour of the wild-type (14028s), rcsB (EG12711), $\mathrm{P}_{r c s D B}$ (MDs1017) and $\mathrm{P}_{r c s B}$ (MDs1018) strains carrying the prcsD plasmid was assayed on LB plates containing $0.4 \%$ agar and IPTG. All images were captured after $12 \mathrm{~h}$ incubation at $37{ }^{\circ} \mathrm{C}$. The images represent one of three independent experiments; the values shown at the foot of each panel correspond to mean values of the independent experiments. 
Taken together, the main conclusion of these results is that the presence of $\mathrm{P}_{r c s B}$ is able to maintain swarming repression at levels similar to those of the wild-type strain, while the $\mathrm{P}_{r c s D B}$ promoter does not, and its role is currently under investigation.

\section{DISCUSSION}

We previously reported that a high level of RcsB regulator inhibits $r c s D$ gene transcription, showing a weak effect on $r c s B$ expression due to the presence of the $\mathrm{P}_{r c s B}$ promoter (Pescaretti et al., 2009). The goal of the present work was to study further the effect of the RcsB regulator on the control of $r c s D$ expression. In addition to the RcsB overproduction reported, $r c s D$ repression also occurs in the $r c s C 11$ mutant and even more so under a physiological condition such as polymyxin $\mathrm{B}$ treatment. With the $\beta$-galactosidase activity and GFP production assays we demonstrated that the $r c s D$ repression induced by a high $\mathrm{RcsB}$ level is due to a specific effect on the $\mathrm{P}_{r c s D B}$ promoter. The $\mathrm{P}_{r c s D B}$ promoter activity was repressed only by the RcsB protein, since no effect was observed in the $r \operatorname{cs} B$ mutant, indicating the RcsBdependence of this effect. Additionally, the identification of a conserved RcsB-binding site on the $\mathrm{P}_{r c s D B}$ promoter confirmed the direct action of the regulator on this promoter, as established by the footprinting assay. No
RcsB-binding site was found in the $\mathrm{P}_{r c s B}$ promoter region. Furthermore, the repression effect was strongly supported by the localization of the RcsB-binding site, overlapping the $\mathrm{P}_{r c s D B}-35$ box described previously (Pescaretti et al., 2009).

Cumulatively, these results suggest that the Rcs system has a mechanism of negative autoregulation. Autoregulation has been observed in other two-component regulatory systems, including the phoPQ operon of Salmonella (Soncini et al., 1995), the phoBR operon of E. coli (Guan et al., 1983), the virA and $\operatorname{virG}$ genes of Agrobacterium tumefaciens (Winans et al., 1994), and the bvgAS operon of Bordetella pertussis (Stibitz \& Miller, 1994). It is important to note that all of the above systems are under a positive autoregulation mechanism. We are describing for what is believed to be the first time that the Rcs system could be negatively autoregulated.

On the basis of our results, a negative autoregulation model for the $S$. Typhimurium Rcs regulatory system is proposed (Fig. 6). In the presence of the signal, the Rcs phosphorelay system is fully activated due to phosphate transfer from RcsC to RcsB, mediated by RcsD. The $r c s B$ gene is expressed from both promoters, $\mathrm{P}_{r c s D B}$ and $\mathrm{P}_{r c s B}$, producing high levels of $\mathrm{RcsB}$, which is then phosphorylated and can modulate the expression of those genes that

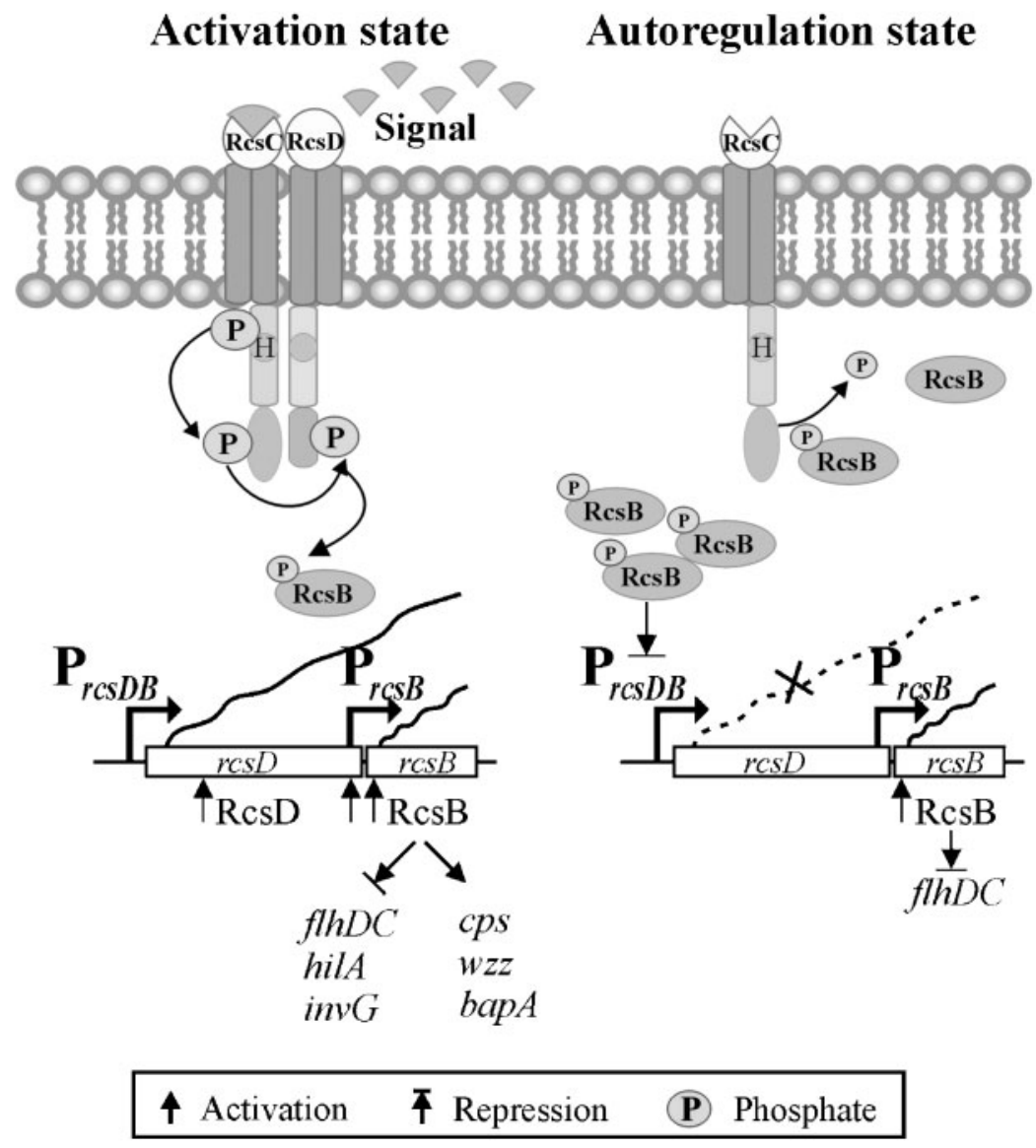

Fig. 6. Negative autoregulation model proposed for the Rcs system. The signal is sensed by RcsC, producing full Rcs system activation, which in turn controls the modulation of the indicated genes (Activation state). After reaching the threshold concentration of RcsB, the $\mathrm{P}_{r C s D B}$ promoter activity is repressed and $r c s B$ expression is maintained at low levels by the $\mathrm{P}_{\text {rcsB }}$ promoter activity (Autoregulation state). 
are required for adaptation (Fig. 6, activation state). After reaching a threshold concentration, RcsB protein represses $r c s D$ transcription by binding to the $\mathrm{P}_{r c s D B}$ promoter. This repression breaks off the Rcs phosphorelay pathway, with the consequent dephosphorylation of RcsB by RcsC phosphatase activity (Fig. 6, autoregulation state). At this point, $r c s B$ expression begins to be controlled only by the $\mathrm{P}_{r c s B}$ promoter, and the RcsB protein returns to basal levels, which are required to maintain the repression of motility.

It has previously been established that activation of the Salmonella Rcs regulatory system interferes with the ability of Salmonella to cause a lethal infection in mice (Mouslim et al., 2004). Furthermore, a close correlation between virulence and flagellar regulation has been reported (Ikeda et al., 2001). In this paper we demonstrate that even when $\mathrm{P}_{r c s D B}$ is quantitatively more active than the $\mathrm{P}_{r c s B}$ promoter, the latter is required to maintain the repression of the RcsB-dependent motility phenotype. The presence of two promoters, $\mathrm{P}_{r c s D B}$ and $\mathrm{P}_{r c s B}$, acquires an important physiological relevance, since it allows the maintenance of bacterial mobility repression even in the negative autoregulation state. Ongoing experiments are being directed toward identifying the physiological signals able to activate the Rcs system and determining how virulence and swarming motility could be influenced.

\section{ACKNOWLEDGEMENTS}

We thank C. Guardia for technical assistance, E. A. Groisman, Washington University School of Medicine, St. Louis, MO, USA, for strains, C. Materon and C. Mouslim for comments on an earlier version of the manuscript, and the PEW Latin American Fellow program for the equipment donated. M. M. P. and F. E. L. are Fellows of the Consejo Nacional de Investigaciones Cientificas y Tecnologicas (CONICET). This work was supported, in part, by FONCYT grant 32124 to M.A.D.; R.D.M. and M.A.D. are Investigators of CONICET.

\section{REFERENCES}

Bader, M. W., Navarre, W. W., Shiau, W., Nikaido, H., Frye, J. G., McClelland, M., Fang, F. C. \& Miller, S. I. (2003). Regulation of Salmonella typhimurium virulence gene expression by cationic antimicrobial peptides. Mol Microbiol 50, 219-230.

Beeston, A. L. \& Surette, M. G. (2002). $p f s$-dependent regulation of autoinducer 2 production in Salmonella enterica serovar Typhimurium. J Bacteriol 184, 3450-3456.

Cano, D. A., Dominguez-Bernal, G., Tierrez, A., Garcia-Del Portillo, F. \& Casadesus, J. (2002). Regulation of capsule synthesis and cell motility in Salmonella enterica by the essential gene igaA. Genetics 162, 1513-1523.

Carballes, F., Bertrand, C., Bouche, J. P. \& Cam, K. (1999). Regulation of Escherichia coli cell division genes ftsA and fts $Z$ by the two-component system $r c s C$-rcsB. Mol Microbiol 34, 442-450.

Chen, M. H., Takeda, S., Yamada, H., Ishii, Y., Yamashino, T. \& Mizuno, T. (2001). Characterization of the $\mathrm{RcsC} \rightarrow \mathrm{YojN} \rightarrow \mathrm{RcsB}$ phosphorelay signaling pathway involved in capsular synthesis in Escherichia coli. Biosci Biotechnol Biochem 65, 2364-2367.
Clarke, D. J., Holland, I. B. \& Jacq, A. (1997). Point mutations in the transmembrane domain of DjlA, a membrane-linked DnaJ-like protein, abolish its function in promoting colanic acid production via the Rcs signal transduction pathway. Mol Microbiol 25, 933-944.

Costa, C. S. \& Anton, D. N. (2001). Role of the ftsAlp promoter in the resistance of mucoid mutants of Salmonella enterica to mecillinam: characterization of a new type of mucoid mutant. FEMS Microbiol Lett 200, 201-205.

Datsenko, K. A. \& Wanner, B. L. (2000). One-step inactivation of chromosomal genes in Escherichia coli K-12 using PCR products. Proc Natl Acad Sci U S A 97, 6640-6645.

Davis, R. W., Bolstein, D. \& Roth, J. R. (1980). Advanced Bacterial Genetics. Cold Spring Harbor, NY: Cold Spring Harbor Laboratory Press.

Delgado, M. A., Mouslim, C. \& Groisman, E. A. (2006). The PmrA/ $\mathrm{PmrB}$ and RcsC/YojN/RcsB systems control expression of the Salmonella O-antigen chain length determinant. Mol Microbiol 60, 39-50.

Erickson, K. D. \& Detweiler, C. S. (2006). The Rcs phosphorelay system is specific to enteric pathogens/commensals and activates $y d e I$, a gene important for persistent Salmonella infection of mice. $\mathrm{Mol}$ Microbiol 62, 883-894.

Ferrières, L. \& Clarke, D. J. (2003). The RcsC sensor kinase is required for normal biofilm formation in Escherichia coli K-12 and controls the expression of a regulon in response to growth on a solid surface. Mol Microbiol 50, 1665-1682.

Fields, P. I., Swanson, R. V., Haidaris, C. G. \& Heffron, F. (1986). Mutants of Salmonella typhimurium that cannot survive within the macrophage are avirulent. Proc Natl Acad Sci U S A 83, 5189-5193.

Francez-Charlot, A., Laugel, B., Van Gemert, A., Dubarry, N., Wiorowski, F., Castanie-Cornet, M. P., Gutierrez, C. \& Cam, K. (2003). RcsCDB His-Asp phosphorelay system negatively regulates the flhDC operon in Escherichia coli. Mol Microbiol 49, 823-832.

Guan, C. D., Wanner, B. \& Inouye, H. (1983). Analysis of regulation of phoB expression using a phoB-cat fusion. J Bacteriol 156, 710-717.

Harshey, R. M. (2003). Bacterial motility on a surface: many ways to a common goal. Annu Rev Microbiol 57, 249-273.

Huang, Y. H., Ferrières, L. \& Clarke, D. J. (2006). The role of the Rcs phosphorelay in Enterobacteriaceae. Res Microbiol 157, 206-212.

Ikeda, J. S., Schmitt, C. K., Darnell, S. C., Watson, P. R., Bispham, J., Wallis, T. S., Weinstein, D. L., Metcalf, E. S., Adams, P. \& other authors (2001). Flagellar phase variation of Salmonella enterica serovar Typhimurium contributes to virulence in the murine typhoid infection model but does not influence Salmonella-induced enteropathogenesis. Infect Immun 69, 3021-3030.

Kalir, S., Mangan, S. \& Alon, U. (2005). A coherent feed-forward loop with a SUM input function prolongs flagella expression in Escherichia coli. Mol Syst Biol 1, 2005.0006.

Kelley, W. L. \& Georgopoulos, C. (1997). Positive control of the twocomponent RcsC/B signal transduction network by DjlA: a member of the DnaJ family of molecular chaperones in Escherichia coli. Mol Microbiol 25, 913-931.

Kim, W. \& Surette, M. G. (2004). Metabolic differentiation in actively swarming Salmonella. Mol Microbiol 54, 702-714.

Majdalani, N. \& Gottesman, S. (2005). The Rcs phosphorelay: a complex signal transduction system. Annu Rev Microbiol 59, 379-405.

Mariscotti, J. F. \& Garcia-del Portillo, F. (2009). Genome expression analyses revealing the modulation of the Salmonella Rcs regulon by the attenuator IgaA. J Bacteriol 191, 1855-1867.

Miller, J. H. (1972). Experiments in Molecular Genetics. Cold Spring Harbor, NY: Cold Spring Harbor Laboratory Press. 
Mouslim, C. \& Groisman, E. A. (2003). Control of the Salmonella ugd gene by three two-component regulatory systems. Mol Microbiol 47, 335-344.

Mouslim, C., Latifi, T. \& Groisman, E. A. (2003). Signal-dependent requirement for the co-activator protein RcsA in transcription of the RcsB-regulated ugd gene. J Biol Chem 278, 50588-50595.

Mouslim, C., Delgado, M. \& Groisman, E. A. (2004). Activation of the RcsC/YojN/RcsB phosphorelay system attenuates Salmonella virulence. Mol Microbiol 54, 386-395.

Pescaretti, M. M., Morero, R. \& Delgado, M. A. (2009). Identification of a new promoter for the response regulator $r c s B$ expression in Salmonella enterica serovar Typhimurium. FEMS Microbiol Lett $\mathbf{3 0 0}$, 165-173.

Ronen, M., Rosenberg, R., Shraiman, B. I. \& Alon, U. (2002). Assigning numbers to the arrows: parameterizing a gene regulation network by using accurate expression kinetics. Proc Natl Acad Sci U S A 99, 10555-10560.

Rosenfeld, N., Elowitz, M. B. \& Alon, U. (2002). Negative autoregulation speeds the response times of transcription networks. J Mol Biol 323, 785-793.

Sambrook, J., Fritsch, E. F. \& Maniatis, T. (1989). Molecular Cloning: a Laboratory Manual. Cold Spring Harbor, NY: Cold Spring Harbor Laboratory Press.

Sanderson, K. E., Hessel, A. \& Rudd, K. E. (1995). Genetic map of Salmonella typhimurium, VIII edition. Microbiol Rev 59, 241-303.

Soncini, F. C., Vescovi, E. G. \& Groisman, E. A. (1995). Transcriptional autoregulation of the Salmonella typhimurium phoPQ operon. J Bacteriol 177, 4364-4371.
Stibitz, S. \& Miller, J. F. (1994). Coordinate regulation of virulence in Bordetella pertussis mediated by the vir (bvg) locus. In Molecular Genetics of Bacterial Pathogenesis, pp. 407-422. Edited by V. L. Miller, J. B. Kaper, D. A. Portney \& R. R. Isberg. Washington, DC: American Society for Microbiology.

Stout, V. \& Gottesman, S. (1990). RcsB and RcsC: a two-component regulator of capsule synthesis in Escherichia coli. J Bacteriol 172, 659669.

Takeda, S., Fujisawa, Y., Matsubara, M., Aiba, H. \& Mizuno, T. (2001). A novel feature of the multistep phosphorelay in Escherichia coli: a revised model of the $\mathrm{RcsC} \rightarrow$ YojN $\rightarrow$ RcsB signalling pathway implicated in capsular synthesis and swarming behaviour. Mol Microbiol 40, 440-450.

Toguchi, A., Siano, M., Burkart, M. \& Harshey, R. M. (2000). Genetics of swarming motility in Salmonella enterica serovar Typhimurium: critical role for lipopolysaccharide. J Bacteriol 182, 6308-6321.

Virlogeux, I., Waxin, H., Ecobichon, C., Lee, J. O. \& Popoff, M. Y. (1996). Characterization of the rcsA and $r c s B$ genes from Salmonella typhi: $r c s B$ through tviA is involved in regulation of $\mathrm{Vi}$ antigen synthesis. J Bacteriol 178, 1691-1698.

Wehland, M. \& Bernhard, F. (2000). The RcsAB box. Characterization of a new operator essential for the regulation of exopolysaccharide biosynthesis in enteric bacteria. J Biol Chem 275, 7013-7020.

Winans, S. C., Mantis, N. J., Chen, C. Y., Chang, C. H. \& Han, D. C. (1994). Host recognition by the VirA, VirG two-component regulatory proteins of Agrobacterium tumefaciens. Res Microbiol 145, 461-473.

Edited by: P. H. Everest 